\title{
Selecting Training method of a rehabilitation robot Based on fuzzy comprehensive evaluation
}

\section{Lei $\mathrm{CHEN}^{1,2,}{ }^{\text {, }}$, Chang-niu YANG ${ }^{1}$, Wen-quan HUANG ${ }^{1}$, Ze-gang SUN ${ }^{1}$ and Yu-cong LIU ${ }^{1}$}

${ }^{1}$ College of Mechanical Engineering

,Sichuan university of Science and Engineering

Zigong city, Sichuan province 643000 , China

${ }^{2}$ Artificial Intelligence Key Laboratory of Sichuan Province

Zigong city, Sichuan province 643000 , China

* Lei CHEN

\begin{abstract}
Keywords: Rehabilitation robot; Training mode; Fuzzy comprehensive evaluation.
Abstract: Gait training is an important part of robotic gait rehabilitation, which is helpful to provide consistent, adjustable physical therapy comparing with traditional manual training. However the selection training mode has become a problem. In order to solve the selection problem of training mode of a lower limb rehabilitation robot, this paper puts forward a method which is based on fuzzy comprehensive evaluation. Firstly, this paper establishes the evaluation model which includes selecting five evaluation indicators, establishing evaluation set, fuzzy evaluation matrix and determining the weight of factors. On the basis, the numerical example is given at the end of this section to illustrate the feasibility of the methodology. The results show that the method is reasonable and effective.
\end{abstract}

\section{Introduction}

It has received extensive attention that the application of robots in the field of medical rehabilitation. Rehabilitation medicine research shows that ${ }^{[1]}$, for the joint function injury patients, been treated with conventional treatment at the same time and complemented by certain rehabilitation training, can make the joint function be improved. Rehabilitation robot is the outcome of the combination of rehabilitation medicine and robot technology. The rehabilitation robot is a kind of medical robot, developed with the motor nerve rehabilitation treatment technology in recent years ${ }^{[2]}$, which has been paid more attention in the field of robot. Fig. 1 shows that the training mode is an important part for patients in rehabilitation process, which can help a patients acquire reasonable treatment based on the current information collection and analysis comparing with standard medical health. The problems of traditional rehabilitation evaluation method are simple measures, processing result simply and single evaluation conclusion $^{[3]}$. Because of human factors, and various evaluation factors, it is difficult to accurately select the proper treatment for patients. So the training mode can't achieve the desire rehabilitation effect, and there is no difference after rehabilitation therapy ${ }^{[4,5]}$, which leads to negative therapy psychology.

In order to improve rehabilitative efficacy, this paper presents a selecting training method of the lower limb rehabilitation robot. Firstly, the rehabilitation evaluation indicator model of training mode is built based on fuzzy comprehensive evaluation method. Secondly, the calculation of the model is implemented, which can automatically select a training mode of a lower limb rehabilitation robot and ensure the scientific treatment for the patient's limb correctly. 


\section{Establishment of evaluation model}

\section{(A) Selections of the evaluation indicator}

Because the selection problem of training mode of the lower limb rehabilitation robot is a kind of fuzzy comprehensive evaluation, this paper chooses five factors, which include the average half step length, age, sex, height and the stability of the lower limb movement and are the basis of selection of rehabilitation mode. Fig. 2 shows the evaluation indicator system of training mode of the lower limb rehabilitation robot. These evaluation indicator are as follows:

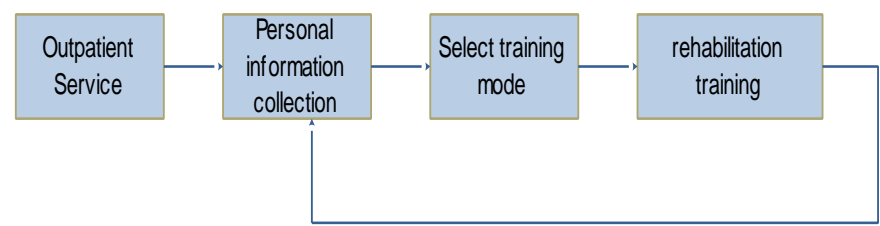

Fig. 1 The process of the rehabilitation training establishment of evaluation model

(1) Average half step length $(\bar{S})$ is half of the length of the single step length which is measured by the 5 gait cycle in the case of no robot assisted walking, and this is an important indicator of the degree of rehabilitation of patients. With consulting literature and clinical experience, it includes six levels: $\mathrm{A}(\bar{S} \leq 0.10) 、 \mathrm{~B}(0.10<\bar{S} \leq 0.20) 、 \mathrm{C}(0.20<\bar{S} \leq 0.30) 、 \mathrm{D}(0.30<\bar{S} \leq 0.40) 、 \mathrm{E}(0.40<\bar{S} \leq 0.50) 、 \mathrm{~F}(0.50<\bar{S})$;

(2)The influence of age is an important factor on the physiological state of the patients in the rehabilitation training which can not be ignored. Patients with the same disease in different age groups have different recovery effects due to their different physiological stages, so the age is a factor. According to the age, there are three stages :under 30s, 30-50years, over 50s;

(3) Different gender of patients with different physical quality, especially in adult patients, male activity, physiological characteristics and female patients with a clear distinction, so gender should also be considered as a factor. The genders are male and female.

(4)Height of the body: less than $1.60 \mathrm{~m}(\mathrm{~L}), 1.60 \mathrm{~m}$ to $1.80 \mathrm{~m}(\mathrm{~N})$, greater than $1.80 \mathrm{~m}(\mathrm{H})$, which respectively is on behalf of the short, medium and high.

(5) The coordination ability of the lower limbs of the human body is a very important index to judge the degree of rehabilitation of the patients and make the rehabilitation plan.

Stability of the lower limb movement is divided into three parts: good, moderate and bad. So the target layer can be divided into five evaluation factors: $u=\left\{u_{1}, u_{2}, u_{3}, u_{4}, u_{5}\right\}$.

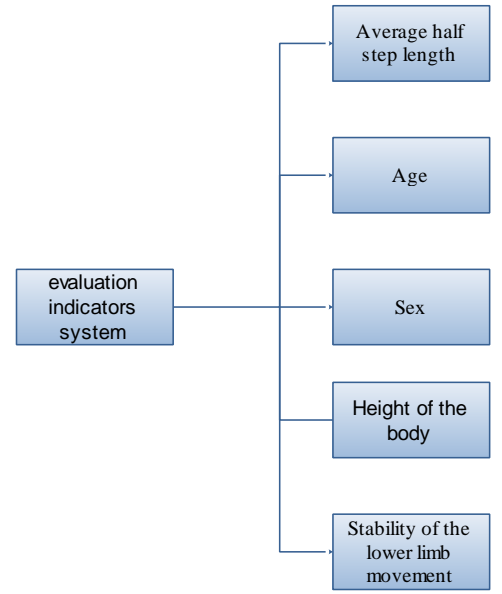

Fig.2 Evaluation indicators system of Training mode

(B) Establishment of evaluation set

On the basis of the evaluation indicator system, a evaluation set of the limb rehabilitation robot was divided into eight fields on fuzzy comprehensive evaluation method:

$$
V=\left\{V_{1}, V_{2}, V_{3}, V_{4}, V_{5}, V_{6}, V_{7}, V_{8}\right\} .
$$

Gait pattern is obtained by gait planning as fig. 3 shows. 
(C) Establishment of fuzzy mapping

Fuzzy mapping of single factor fuzzy evaluation:

$$
\begin{gathered}
f: u-V \\
u_{i} \rightarrow f\left(u_{i}\right) \square\left(r_{i 1}, r_{i 2}, r_{i 3}, r_{i 4}, r_{i 5}, r_{i 6}, r_{i 7}, r_{i 8}\right)
\end{gathered}
$$

The fuzzy evaluation set of the factors on the choice of gait rehabilitation mode is $f\left(u_{i}\right)$; $r_{i j}(i=1,2,3,4,5 ; j=1,2,3,4,5,6,7,8)$ is a membership of the rehabilitation mode.

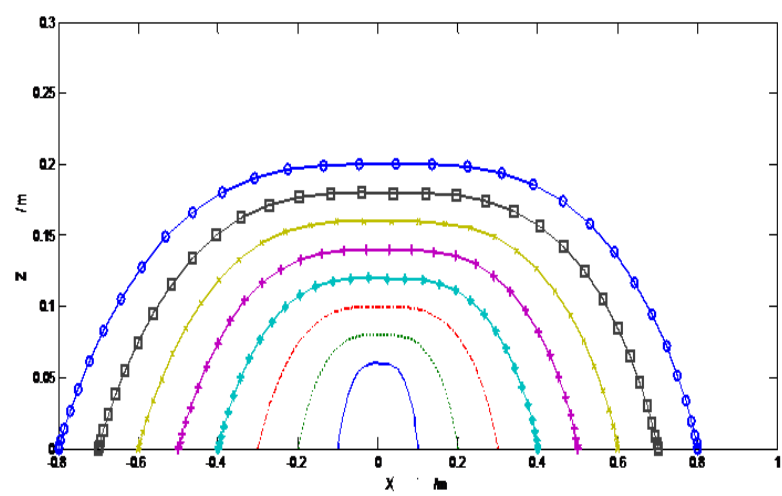

Fig.3 Eight short-term rehabilitation training patterns

(D) Establishment of fuzzy evaluation matrix

Fuzzy evaluation matrix is established by taking into the indicators to rehabilitation mode of lower limb rehabilitation robot. So the evaluation matrix of rehabilitation mode is as follows:

$$
R=\left[\begin{array}{l}
f\left(u_{1}\right) \\
f\left(u_{2}\right) \\
f\left(u_{3}\right) \\
f\left(u_{4}\right) \\
f\left(u_{5}\right)
\end{array}\right]=\left[\begin{array}{llllllll}
r_{11} & r_{12} & r_{13} & r_{14} & r_{15} & r_{16} & r_{17} & r_{18} \\
r_{21} & r_{22} & r_{23} & r_{24} & r_{25} & r_{26} & r_{27} & r_{28} \\
r_{31} & r_{32} & r_{33} & r_{34} & r_{35} & r_{36} & r_{37} & r_{38} \\
r_{41} & r_{42} & r_{43} & r_{44} & r_{45} & r_{46} & r_{47} & r_{48} \\
r_{51} & r_{52} & r_{53} & r_{54} & r_{55} & r_{56} & r_{57} & r_{58}
\end{array}\right]
$$

Because ${ }^{u_{1}}$ has six factor levels, ${ }^{u_{2}}$ has three factor levels, ${ }^{u_{3}}$ has two factors, ${ }^{u_{4}}$ has two factors, $u_{5}$ has two factors, rehabilitation mode has $324^{(6 \times 3 \times 2 \times 3 \times 3)}$ fuzzy evaluation matrixes.

(E) Determination of the weights of evaluation factors at different levels

For the choice of the human lower limb rehabilitation training mode, the average half step is the most important, followed by the stability of lower limb activity, and then the height, age, sex and the five elements. Therefore, we should assign different weights on the composition of the weight set for:

$$
w=\left(\frac{0.35}{u_{1}}, \frac{0.15}{u_{2}}, \frac{0.1}{u_{3}}, \frac{0.15}{u_{4}}, \frac{0.25}{u_{5}}\right)
$$

So the aid of expert experience is needed, and to assign various indicators is by the analytic hierarchy process, as shown in Table 1.Using the data in table 1 by the improved method ${ }^{[6]}$, the relative weight of each purpose component indicator weight can be directly calculated. In order to reflect the actual situation of the lower limb rehabilitation robot rehabilitation evaluation, the weighted average model of fuzzy comprehensive evaluation ${ }^{[7]}$ is chosen. Expression is:

$$
B=w \circ R=\left(b_{1}, b_{2}, b_{3}, b_{4}, b_{5}, b_{6}, b_{7}, b_{8}\right)
$$

The selection of the synthetic operation is also very important, considering that the number of factors affecting the target in this paper is less, and the average half step factor is in the dominant position, so the model is used to synthesize the operation. Expression is:

$$
b_{j}=\sum_{i=1}^{5} w_{i} \square r_{i j}(j=1,2,3,4,5,6,7,8)
$$


(F) Determination of the weights of evaluation factors at different levels

A fuzzy statistical method is adopted based on the expert investigation. On the basis of evaluation objects and the evaluation indicator, the experts determine the membership degree of each evaluation factor and single factor evaluation matrix is obtained.

$$
\begin{gathered}
\text { Membership }=\frac{\text { the number of evaluation experts }}{\text { The total number of eligible experts }} \\
\text { Tab.1 Membership of the influence factors }
\end{gathered}
$$

\begin{tabular}{|c|c|c|c|c|c|c|c|c|c|}
\hline \multirow{2}{*}{\multicolumn{2}{|c|}{ Factors }} & \multicolumn{8}{|c|}{ Membership of training mode } \\
\hline & & \multirow{2}{*}{$\begin{array}{l}V_{1} \\
0.8 \\
5\end{array}$} & \multirow{2}{*}{$\begin{array}{l}V_{2} \\
0.8\end{array}$} & \multirow{2}{*}{$\begin{array}{l}V_{3} \\
0.6\end{array}$} & \multirow{2}{*}{$\begin{array}{l}V_{4} \\
0.4\end{array}$} & \multirow{2}{*}{$\begin{array}{l}V_{5} \\
0\end{array}$} & \multirow{2}{*}{$\begin{array}{l}V_{6} \\
0\end{array}$} & \multirow{2}{*}{$\begin{array}{l}V_{7} \\
0\end{array}$} & \multirow{2}{*}{$\frac{V_{8}}{0}$} \\
\hline \multirow{6}{*}{$\begin{array}{l}\text { Average } \\
\text { half step } \\
\text { length }\end{array}$} & A & & & & & & & & \\
\hline & B & 0.7 & 0.8 & 0.75 & 0.6 & 0.5 & 0 & 0 & 0 \\
\hline & $\mathrm{C}$ & 0.7 & 0.8 & 0.85 & 0.8 & 0.6 & 0 & 0 & 0 \\
\hline & $\mathrm{D}$ & 0.6 & 0.7 & 0.8 & 0.85 & 0.8 & 0.5 & 0 & 0 \\
\hline & $\mathrm{E}$ & 0.4 & 0.6 & 0.7 & 0.75 & 0.8 & 0.85 & 0.8 & 0.7 \\
\hline & $\mathrm{F}$ & 0 & 0 & 0 & 0.7 & 0.75 & 0.85 & 0.85 & 0.8 \\
\hline \multirow{3}{*}{ Age } & Age1 & 0 & 0 & 0 & 0 & 0.65 & 0.75 & 0.8 & 0.6 \\
\hline & Age2 & 0 & 0 & 0 & 0 & 0.70 & 0.80 & 0.75 & 0.6 \\
\hline & Age3 & 0 & 0 & 0.4 & 0.7 & 0.85 & 0.75 & 0.6 & 0 \\
\hline \multirow{2}{*}{ Sex } & M & 0 & 0 & 0 & 0.55 & 0.7 & 0.8 & 0.85 & 0.75 \\
\hline & F & 0 & 0 & 0 & 0.45 & 0.75 & 0.85 & 0.80 & 0.6 \\
\hline \multirow{3}{*}{ Height } & $\mathrm{L}$ & 0.7 & 0.85 & 0.75 & 0.4 & 0 & 0 & 0 & 0 \\
\hline & $\mathrm{N}$ & 0.4 & 0.7 & 0.8 & 0.6 & 0.3 & 0 & 0 & 0 \\
\hline & $\mathrm{H}$ & 0 & 0.5 & 0.7 & 0.85 & 0.5 & 0.3 & 0 & 0 \\
\hline \multirow{3}{*}{ Stability } & Good & 0 & 0.4 & 0.7 & 0.8 & 0.6 & 0.4 & 0 & 0 \\
\hline & Moderate & 0.4 & 0.7 & 0.85 & 0.65 & 0.4 & 0 & 0 & 0 \\
\hline & $\mathrm{Bad}$ & 0.7 & 0.8 & 0.6 & 0.4 & 0 & 0 & 0 & 0 \\
\hline
\end{tabular}

\section{Example analysis}

Tab. 2 shows statistics of personal information of five patients with lower limb movement disorders with different prevalence of stroke.The judgment matrix can be calculated by the introduced method. This paper takes No.1 patient as a example. The evaluation indicator:

$$
u=\left(u_{1}, u_{2}, u_{3}, u_{4}, u_{5}\right)=(B, \text { Agel }, M, H, \text { Moderate })
$$

The evaluation set:

$$
V=\left(V_{1}, V_{2}, V_{3}, V_{4}, V_{5}, V_{6}, V_{7}, V_{8}\right)
$$

The judgment matrix :

$$
R=\left[\begin{array}{l}
f\left(u_{1}\right) \\
f\left(u_{2}\right) \\
f\left(u_{3}\right) \\
f\left(u_{4}\right) \\
f\left(u_{5}\right)
\end{array}\right]=\left[\begin{array}{cccccccc}
0.7 & 0.8 & 0.75 & 0.6 & 0.5 & 0 & 0 & 0 \\
0 & 0 & 0 & 0 & 0.65 & 0.75 & 0.8 & 0.6 \\
0 & 0 & 0 & 0.55 & 0.7 & 0.8 & 0.85 & 0.75 \\
0 & 0.5 & 0.7 & 0.85 & 0.5 & 0.3 & 0 & 0 \\
0.4 & 0.7 & 0.85 & 0.6 & 0.4 & 0 & 0 & 0
\end{array}\right]
$$

The weights of the factors:

$$
w=(0.35,0.15,0.1,0.15,0.25)
$$

Combined with the weight, then: 


$$
\begin{aligned}
& B=w \circ R=\left(b_{1}, b_{2}, b_{3}, b_{4}, b_{5}, b_{6}, b_{7}, b_{8}\right) \\
&=\left[\begin{array}{c}
0.35 \\
0.15 \\
0.1 \\
0.15 \\
0.25
\end{array}\right]^{T}\left[\begin{array}{cccccccc}
0.7 & 0.8 & 0.75 & 0.6 & 0.5 & 0 & 0 & 0 \\
0 & 0 & 0 & 0 & 0.65 & 0.75 & 0.8 & 0.6 \\
0 & 0 & 0 & 0.55 & 0.7 & 0.8 & 0.85 & 0.75 \\
0 & 0.5 & 0.7 & 0.85 & 0.5 & 0.3 & 0 & 0 \\
0.4 & 0.7 & 0.85 & 0.6 & 0.4 & 0 & 0 & 0
\end{array}\right] \\
&=(0.345,0.53,0.58,0.5425,0.5175,0.2375,0.205,0.165)
\end{aligned}
$$

In conclusion, rehabilitation evaluation judgment matrix is:

$$
b_{3}=\operatorname{Max}\left(b_{1}, b_{2}, b_{3}, b_{4}, b_{5}, b_{6}, b_{7}, b_{8}\right)=0.58
$$

\begin{tabular}{|c|c|c|c|c|c|}
\hline \multirow[b]{2}{*}{ Patient } & \multicolumn{5}{|c|}{ Information } \\
\hline & $\begin{array}{l}\text { Average } \\
\text { half step } \\
\text { length }(\mathrm{m})\end{array}$ & Age & Gender & $\begin{array}{l}\text { Height } \\
\text { (m) }\end{array}$ & Stability \\
\hline 1 & 0.18 & 20 & M & 1.85 & Moderate \\
\hline 2 & 0.35 & 27 & F & 1.74 & Good \\
\hline 3 & 0.07 & 40 & M & 1.55 & $\mathrm{Bad}$ \\
\hline 4 & 0.16 & 35 & F & 1.82 & Moderate \\
\hline 5 & 0.25 & 50 & $\mathrm{M}$ & 1.58 & $\mathrm{Bad}$ \\
\hline
\end{tabular}

Tab.2 The information of five patients

Tab.3 The selection of the rehabilitation patterns for patients

\begin{tabular}{|c|c|c|c|c|c|}
\hline Patient & 1 & 2 & 3 & 4 & 5 \\
\hline Training Mode & $V_{3}$ & $V_{5}$ & $V_{2}$ & $V_{3}$ & $V_{2}$ \\
\hline
\end{tabular}

Because the rehabilitation status is general with the maximum membership degree method, the rehabilitation evaluation result is 0.58 . So rehabilitation training mode is $V_{3}$, and the patient's limb will recover gradually and presents a step by step, until achieve medical health standard. Therefore, selecting the other training modes is like this. The results are shown in Tab.3.

\section{Conclusions}

Example analysis shows that the established fuzzy evaluation model of selecting training mode is objectively feasibility, which can put forward a better way for patients in rehabilitation training and provide the theoretical basis for the realization of the next control system. Comparing with traditional selecting rehabilitation training mode, the established model of training mode will be an important for the future development of rehabilitation robots, which will improve the patient's quality of rehabilitation training.

\section{Acknowledgement}

This research was financially supported by the Fund of Artificial Intelligence Key Laboratory of Sichuan Province ( No.2014RYJ04). 


\section{References}

[1] Ge Yuping, Discussion nursing and rehabilitation on fracture and joint injury, Health Vocational Education(2006).

[2] Lump, Burgar G. The MIME robotic system for upper-limb neuron-rehabilitation: results from a clinical trial insubacute stroke. In: Proceedings of the 9th International Conference on Rehabilitation Robotics, Chicago, USA, 2005, pp.511-514.

[3] QinYin, BiSheng, WangFu-gen.Upper limb function commonly used evaluation methods and clinical application of cerebral apoplexy. Chinese Journal of Rehabilitation.Medicine.Vol.19, No.3, 2001, pp.232-233.

[4] Qiu Dong. Multi-indicator comprehensive evaluation method of system analysis. Beijing: China statistics press, 1991.

[5] Burgar C G, Lum P S. ShorPC. Development of Robots for RehabilitationTherapy: The aloAltoVA/Stanford,Experience.JournalRehabilitationResearchDevelopment.Vol.37, No.6,2000, pp. 663-673.

[6] Zou Zhi-yong. Engineering machinery maintenance status quo and countermeasure study. Xi 'an: chang 'an university, 2002.

[7] Huang Hong-zhong. Mechanical design fuzzy optimization theory and application. Science press, 1997. 